\title{
Surface-Enhanced Raman Spectroscopy Detection of Ricin B Chain in Human Blood
}

\author{
Antonio R Campos, ${ }^{1}$ Zhe Gao, ${ }^{1}$ Marty Blaber, ${ }^{2}$ Rong Zhuang, ${ }^{2}$ George Schatz, ${ }^{2}$ \\ Richard P. Van Duyne ${ }^{2}$ and Christy L. Haynes ${ }^{1 *}$ \\ ${ }^{1}$ Department of Chemistry, University of Minnesota, Twin Cities, 207 Pleasant Street \\ SE, Minneapolis, Minnesota 55455, United States \\ ${ }^{2}$ Department of Chemistry, Northwestern University, 2145 Sheridan Rd, Evanston, \\ Illinois 60208, United States
}

Author to whom correspondence should be addressed: chaynes@umn.edu Supporting Information (SI) -computational modeling of aptamers, characterization of ricin B chain by SAMDI, non-specific bonding of BSA and stability of AgFONs

\section{Ricin B chain aptamer}

The sequence of the ricin B chain aptamer is 5' ACA CCC ACC GCA GGC AGA CGC AAC GCC TCG GAG ACT AGC C 3'.The folding pattern of this aptamer was predicted by Lamont et al; ${ }^{1}$ it contains a bulge loop (G10-C29) and a hairpin loop $(\mathrm{C} 15-\mathrm{G} 25)$. 

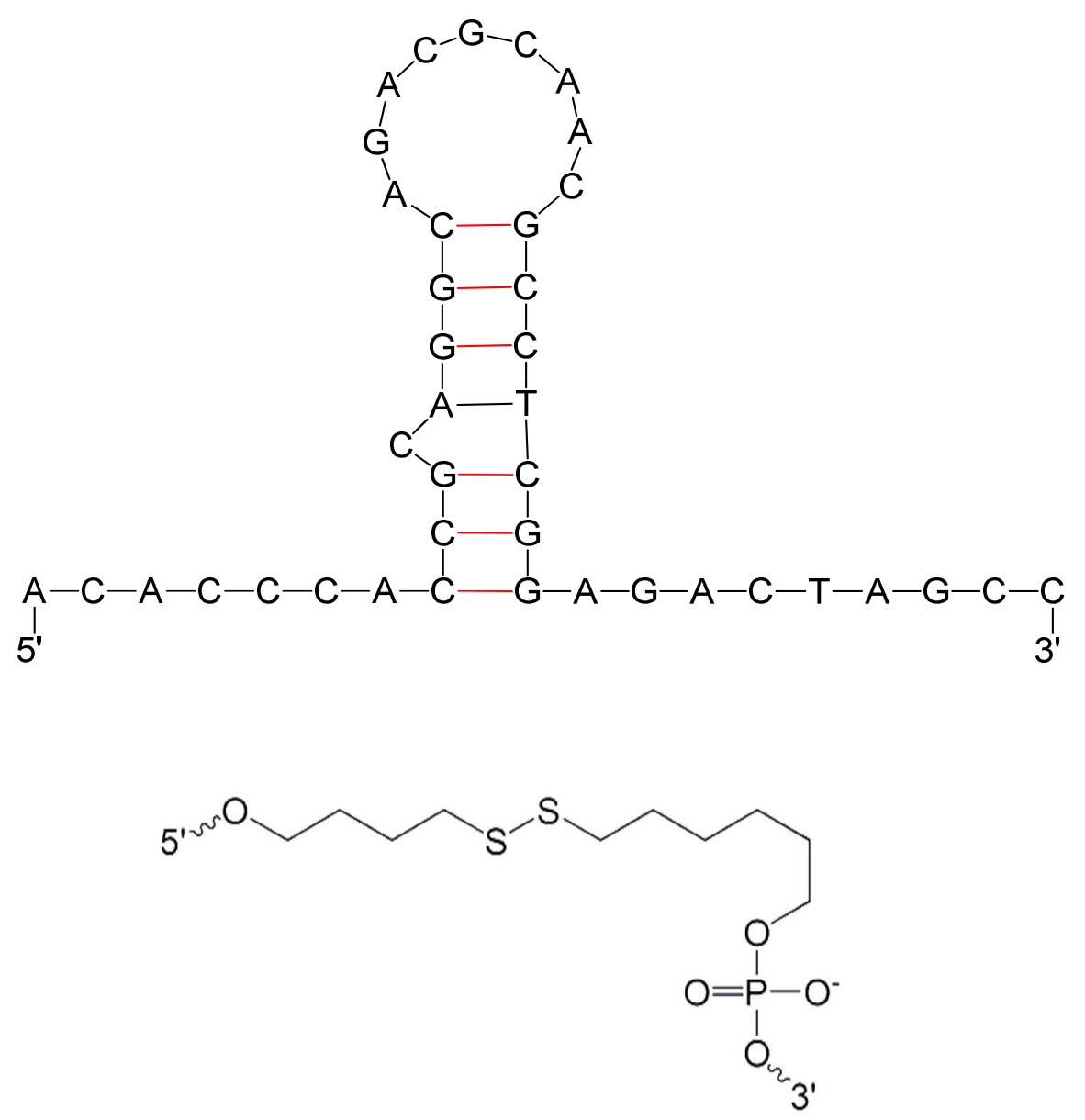

Figure S1. Folding of the ricin B chain aptamer adapted from Lamont et al and the structure of the spacer moiety between the dithiol functionality and the DNA.

\section{Stability of AgFON-BT in blood for 3 or 10 days}

To assess multi-day stability of the AgFONs in human whole blood, a study was initiated with a standard SERS reporter molecule rather than the more expensive aptamer since both of the molecules are connected on the AgFON via Ag-S bond. A 
AgFON was functionalized with benzenethiol (1 $\mathrm{mM}$ in ethanol, overnight, ) and the

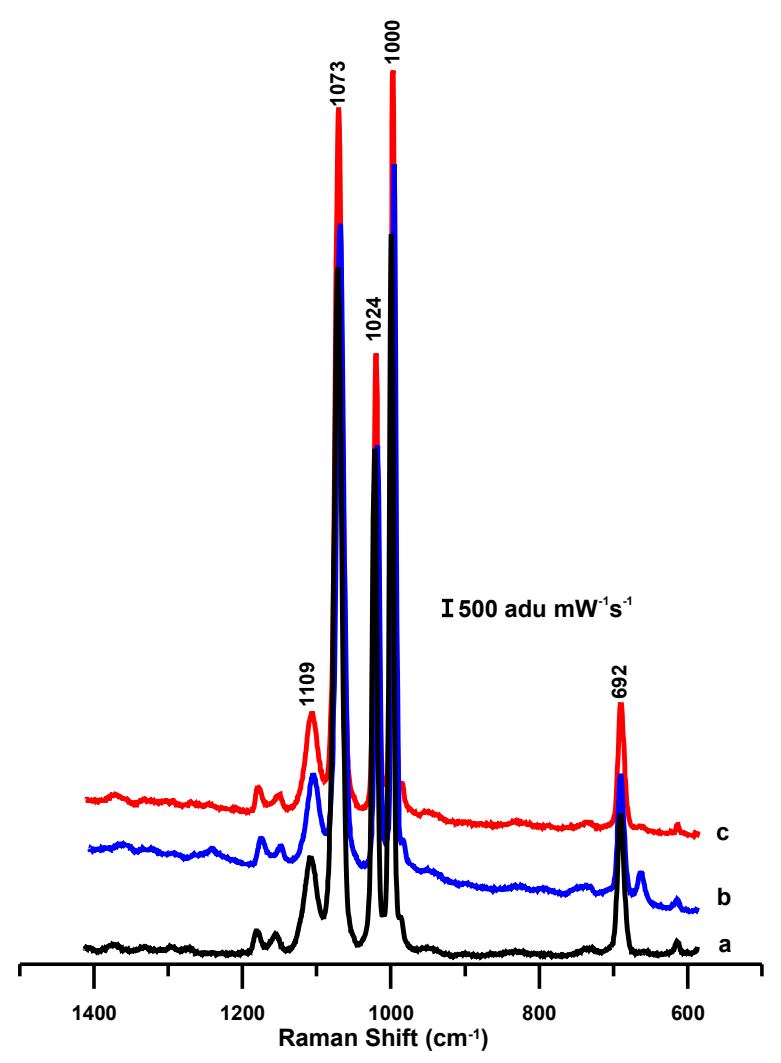

Figure S2. SERS of benzenethiol on a AgFON in human blood for (a) 0 days, (b) 3 days, and (c) 10 days. Each presented spectrum is an average of 7 spectra acquired from 7 randomly selected spots on a single substrate. Spectra are artificially offset vertically for clarity.

spectrum of the functionalized surface was acquired followed by incubation in blood.

\section{Computational modeling of aptamers with respect to the distance} dependence of surface-enhanced Raman spectroscopy 
For the experiments detailed herein, a spacer (shown at the bottom of Figure S1) was implemented to achieve good orientation of the aptamer on the substrate. Assuming that the aptamer was perpendicular to the plasmonic substrate, the vibrational signatures of the first seven bases were included in all calculations based on their proximity to generated electromagnetic fields, while the distances of the other bases were considered in one of two ways. The intensity of the spectrum can be expressed using two equations:

$$
\begin{aligned}
& I=\left(\frac{a+r}{a}\right)^{-10} \\
& I=\sum_{i} I_{i} \exp \left(-\frac{d_{i}}{D}\right)
\end{aligned}
$$

where a is the radius of curvature of the roughness feature on the silver surface, $I$ is the intensity of Raman mode, $I_{i}$ denotes the Raman intensity of each base, $d_{i}$ is the distance of each base to the surface, and D is the damping parameter. Here we choose D to be $1.22 \mathrm{~nm}^{2}$ And the distance between bases is about $2.2 \AA{ }^{3}$ The two possible scenarios considered were:

(1) All bases beyond the $7^{\text {th }}$ are considered to be the same distance from the surface. In this case, the intensity can be expressed as: 


$$
\begin{aligned}
& I=I_{A}\left[1+\exp \left(-\frac{4.4}{12.2}\right)+\exp \left(-\frac{13.2}{12.2}\right)\right]+I_{c}\left[\exp \left(-\frac{2.2}{12.2}\right)+\exp \left(-\frac{6.6}{12.2}\right)+\exp \left(-\frac{8.8}{12.2}\right)+\exp \left(-\frac{11}{12.2}\right)\right] \\
& +\left(8 I_{A}+10 I_{G}+13 I_{c}+2 I_{T}\right) \exp \left(-\frac{15.4}{12.2}\right) \\
& =I_{A}\left[1+\exp \left(-\frac{4.4}{12.2}\right)+\exp \left(-\frac{13.2}{12.2}\right)+8 \exp \left(-\frac{15.4}{12.2}\right)\right]+I_{C}\left[\begin{array}{l}
\exp \left(-\frac{2.2}{12.2}\right)+\exp \left(-\frac{6.6}{12.2}\right)+\exp \left(-\frac{8.8}{12.2}\right) \\
+\exp \left(-\frac{11}{12.2}\right)+13 \exp \left(-\frac{15.4}{12.2}\right)
\end{array}\right] \\
& +10 I_{G} \exp \left(-\frac{15.4}{12.2}\right)+2 I_{T} \exp \left(-\frac{15.4}{12.2}\right)
\end{aligned}
$$

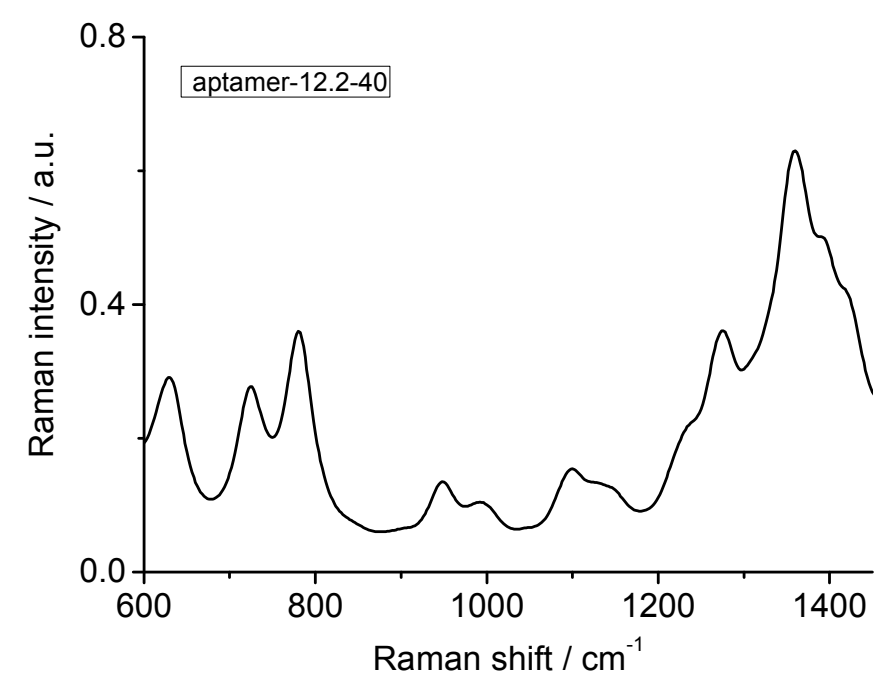

Figure S3. Theoretical SERS spectrum of ricin B chain aptamer in the first scenario, where all bases beyond the $7^{\text {th }}$ are assumed to contribute equally.

(2) All bases beyond the $7^{\text {th }}$ nucleotide are too far away from the surface to contribute significantly to the SERS intensity. In this case, the intensity can be expressed as: 


$$
\begin{aligned}
& I=I_{A}\left[1+\exp \left(-\frac{4.4}{12.2}\right)+\exp \left(-\frac{13.2}{12.2}\right)\right]+I_{c}\left[\exp \left(-\frac{2.2}{12.2}\right)+\exp \left(-\frac{6.6}{12.2}\right)+\exp \left(-\frac{8.8}{12.2}\right)+\exp \left(-\frac{11}{12.2}\right)\right] \\
& +\left(I_{A}+I_{G}+I_{c}\right) \exp \left(-\frac{15.4}{12.2}\right)+\left(2 I_{G}+I_{c}\right) \exp \left(-\frac{17.6}{12.2}\right)+\left(I_{A}+I_{G}+I_{c}\right) \exp \left(-\frac{19.8}{12.2}\right)+I_{c} \exp \left(-\frac{20.9}{12.2}\right) \\
& +\left(I_{A}+I_{T}+I_{c}\right) \exp \left(-\frac{22}{12.2}\right)+\left(I_{T}+I_{G}+I_{c}\right) \exp \left(-\frac{24.2}{12.2}\right)+\left(I_{A}+I_{G}+I_{c}\right) \exp \left(-\frac{26.4}{12.2}\right) \\
& +\left(2 I_{G}+I_{c}\right) \exp \left(-\frac{28.6}{12.2}\right)+\left(I_{A}+I_{c}\right) \exp \left(-\frac{29.7}{12.2}\right)+\left(I_{A}+I_{G}+I_{c}\right) \exp \left(-\frac{30.8}{12.2}\right)+2 I_{A} \exp \left(-\frac{31.9}{12.2}\right) \\
& +3 I_{C} \exp \left(-\frac{33}{12.2}\right)+I_{G} \exp \left(-\frac{34.1}{12.2}\right) \\
& =I_{A}\left[\begin{array}{l}
1+\exp \left(-\frac{4.4}{12.2}\right)+\exp \left(-\frac{13.2}{12.2}\right)+\exp \left(-\frac{15.4}{12.2}\right)+\exp \left(-\frac{19.8}{12.2}\right)+\exp \left(-\frac{22}{12.2}\right)+\exp \left(-\frac{26.4}{12.2}\right) \\
+\exp \left(-\frac{29.7}{12.2}\right)+\exp \left(-\frac{30.8}{12.2}\right)+2 \exp \left(-\frac{31.9}{12.2}\right)
\end{array}\right] \\
& +I_{c}\left[\begin{array}{l}
\exp \left(-\frac{2.2}{12.2}\right)+\exp \left(-\frac{6.6}{12.2}\right)+\exp \left(-\frac{8.8}{12.2}\right)+\exp \left(-\frac{11}{12.2}\right)+\exp \left(-\frac{15.4}{12.2}\right)+\exp \left(-\frac{17.6}{12.2}\right) \\
+\exp \left(-\frac{19.8}{12.2}\right)+\exp \left(-\frac{20.9}{12.2}\right)+\exp \left(-\frac{22}{12.2}\right)+\exp \left(-\frac{24.2}{12.2}\right)+\exp \left(-\frac{26.4}{12.2}\right)+\exp \left(-\frac{28.6}{12.2}\right) \\
+\exp \left(-\frac{29.7}{12.2}\right)+\exp \left(-\frac{30.8}{12.2}\right)+3 \exp \left(-\frac{33}{12.2}\right)
\end{array}\right] \\
& +I_{G}\left[\begin{array}{l}
\exp \left(-\frac{15.4}{12.2}\right)+2 \exp \left(-\frac{17.6}{12.2}\right)+\exp \left(-\frac{19.8}{12.2}\right)+\exp \left(-\frac{24.2}{12.2}\right)+\exp \left(-\frac{26.4}{12.2}\right)+2 \exp \left(-\frac{28.6}{12.2}\right) \\
+\exp \left(-\frac{30.8}{12.2}\right)+\exp \left(-\frac{34.1}{12.2}\right)
\end{array}\right] \\
& +I_{T}\left[\exp \left(-\frac{22}{12.2}\right)+\exp \left(-\frac{24.2}{12.2}\right)\right]
\end{aligned}
$$




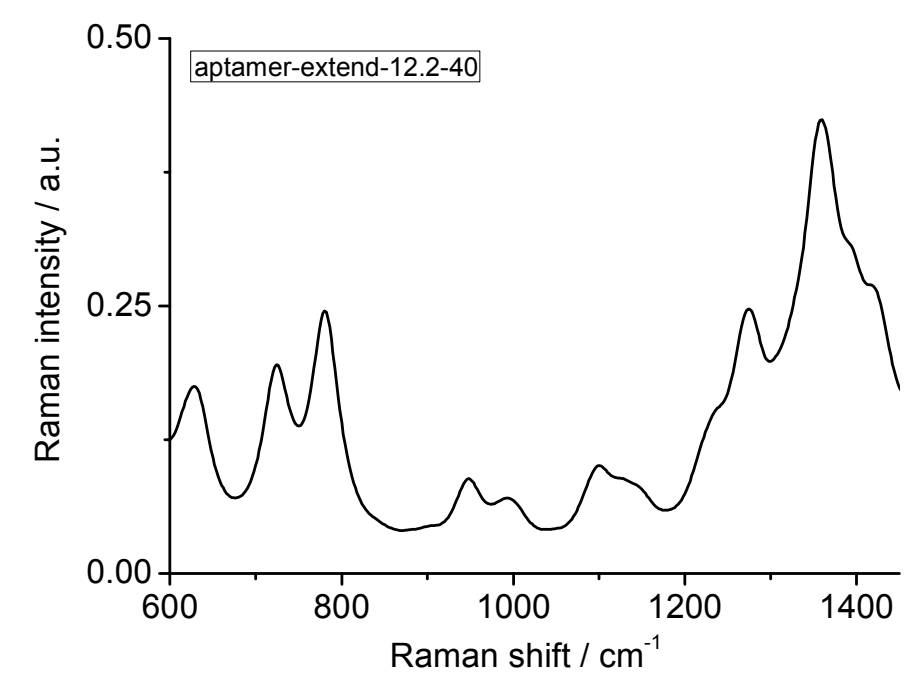

Figure S4. Theoretical SERS spectrum of ricin B chain aptamer in the second scenario where all DNA bases beyond the 7 th are assumed not to contribute to the SERS intensity.

\section{Characterization of ricin B chain on aptamer-modified AgFONs by SAMDI}

Self-assembled monolayer matrix-assisted laser desorption ionization mass spectrometry (SAMDI MS) was used to corroborate the presence of Ricin B chain on aptamer-functionalized AgFONs. Measurements were performed using an Applied Biosystems $^{\odot} 4800$ MALDI TOF TOF mass spectrometer. A 355-nm Nd:YAG laser was used as the desorption/ionization source. The ricin B chain spectra were collected in the high mass linear mode using sinapic acid (Sigma-Aldrich, Inc.; $10 \mathrm{mg} / \mathrm{mL}$ in acetone) as the matrix. Each spectrum was the average of 800 shots and Data Explorer $\left(\right.$ Applied Biosystems $^{\odot}$ ) software was used for analysis. The SAMDI spectrum shows a 
peak at $32 \mathrm{kDa}$ corresponding to the intact Ricin B chain with a mass-to-charge $(\mathrm{m} / \mathrm{z})$ ratio of +1 as well as two peaks around $16 \mathrm{kDa}$ corresponding to an $\mathrm{m} / \mathrm{z}$ ratio of +2 . Given that Ricin B chain has multiple N-glycosylation sites, it is likely that the two different peaks correspond to different glycosylation states. ${ }^{4}$

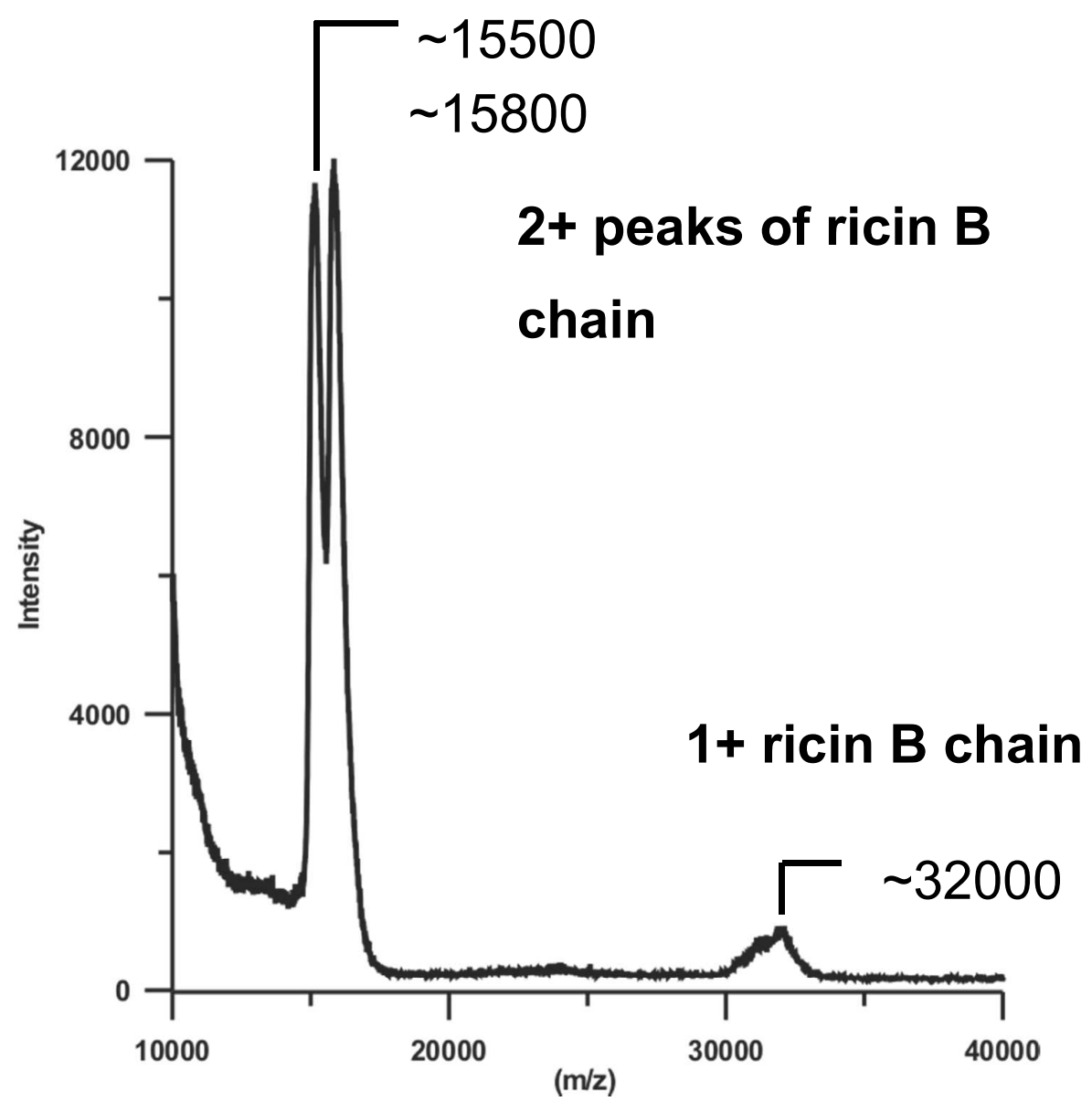

Figure S5. Self-assembled monolayer matrix-assisted laser desorption-ionization MS (SAMDI MS) indicates that ricin B chain is present on the aptamer-modified AgFON surface, corroborating SERS data. 


\section{Stability of AgFON-apt in blood for 10 days and its ability to detect RBC}

\section{after 10 day exposure to blood}

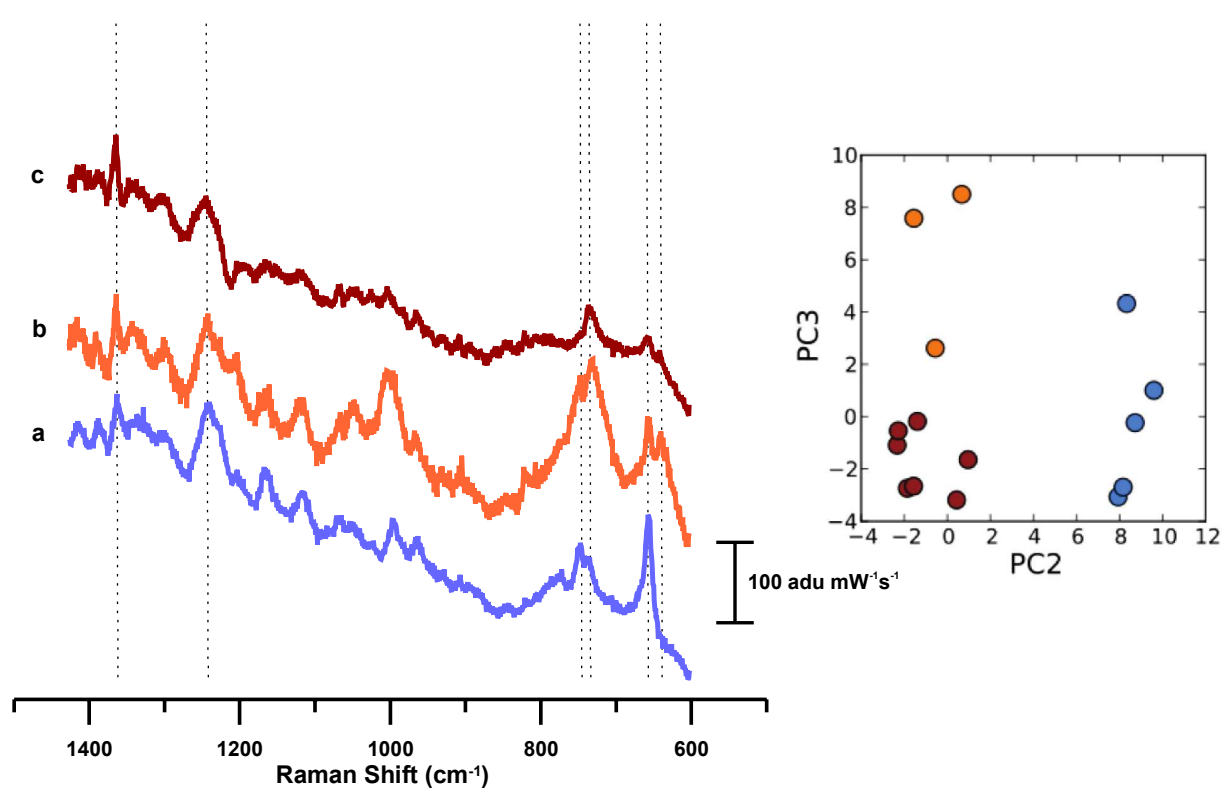

Figure S6. SERS on a AgFON of (a) nuclease-resistant ricin B chain aptamer in blood for 10 days (b) nuclease-resistant ricin B chain aptamer in whole-human blood for 10 days followed by ricin B chain exposure (c) AgFON in human whole blood for 10 days. Spectra are artificially offset in the vertical direction for clarity. On the right, PCA plot shows grouping of the nuclease-resistant aptamer (blue dots), nuclease-resistant aptamer in blood (orange dots), and nuclease-resistant aptamer in blood after exposure to ricin B chain (red dots).

\section{Non-specific binding of BSA on ricin B chain aptamer modified AgFONs}

To confirm the specificity of binding of ricin B chain to the ricin B chain aptamer, BSA, a major protein in blood, was exposed to the aptamer-AgFONs as a potential 
non-specific binding agent. The resulting SERS spectra (figure S7) were analyzed by PCA. The spectra for BSA-exposed aptamer-modified AgFONs cluster with those of aptamer-modified AgFONs, suggesting that BSA does not have affinity for the ricin B chain aptamer (figure S8). 


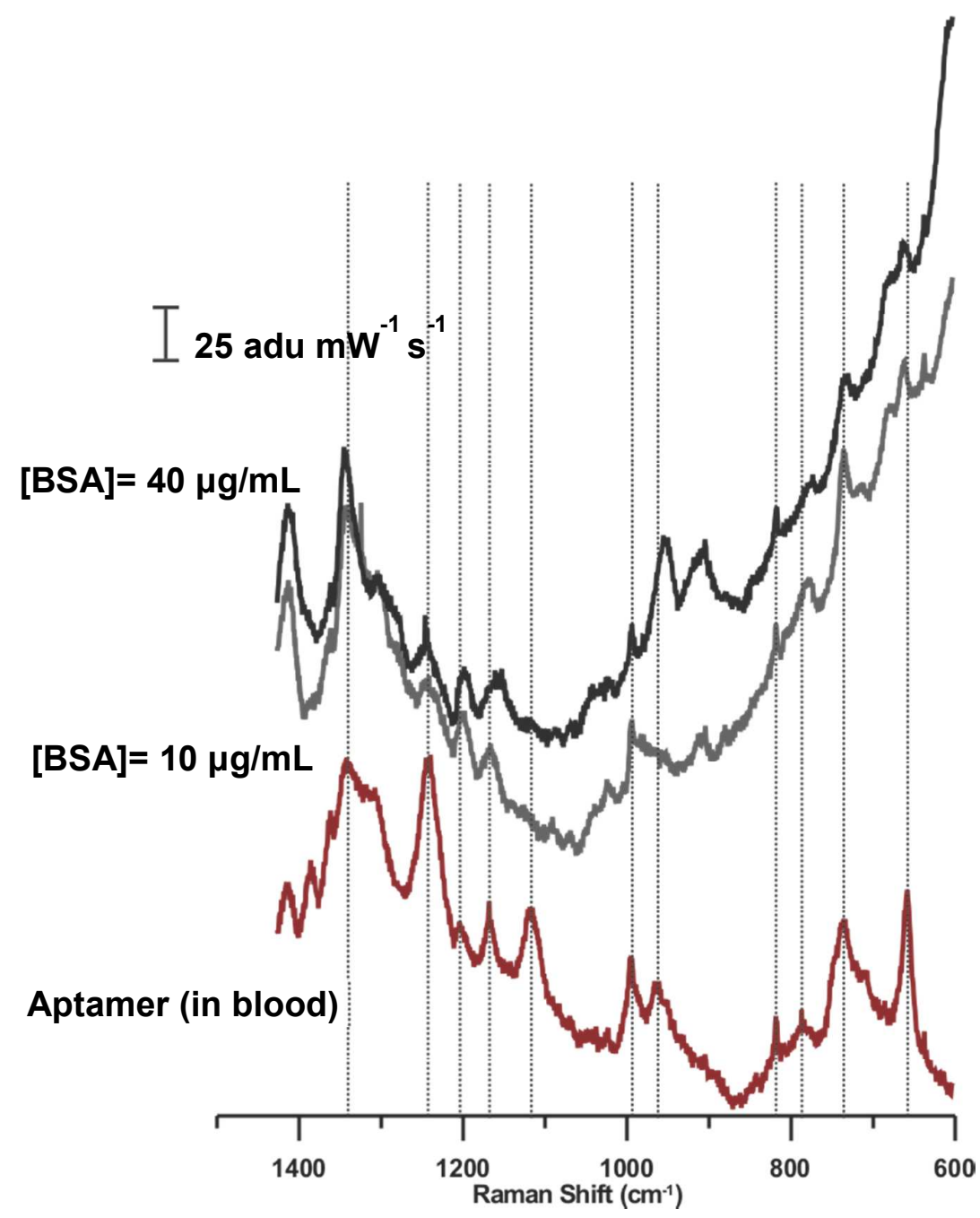

Figure S7. SERS on aptamer-modified AgFONs exposed to various concentrations of BSA in blood. Spectra are vertically offset for clarity, $\lambda_{\mathrm{ex}}=632.8 \mathrm{~nm}$. Each spectrum is the average of 7 randomly chosen spots on the AgFON. PCA of this data is shown in figure $\mathrm{S} 8$. 

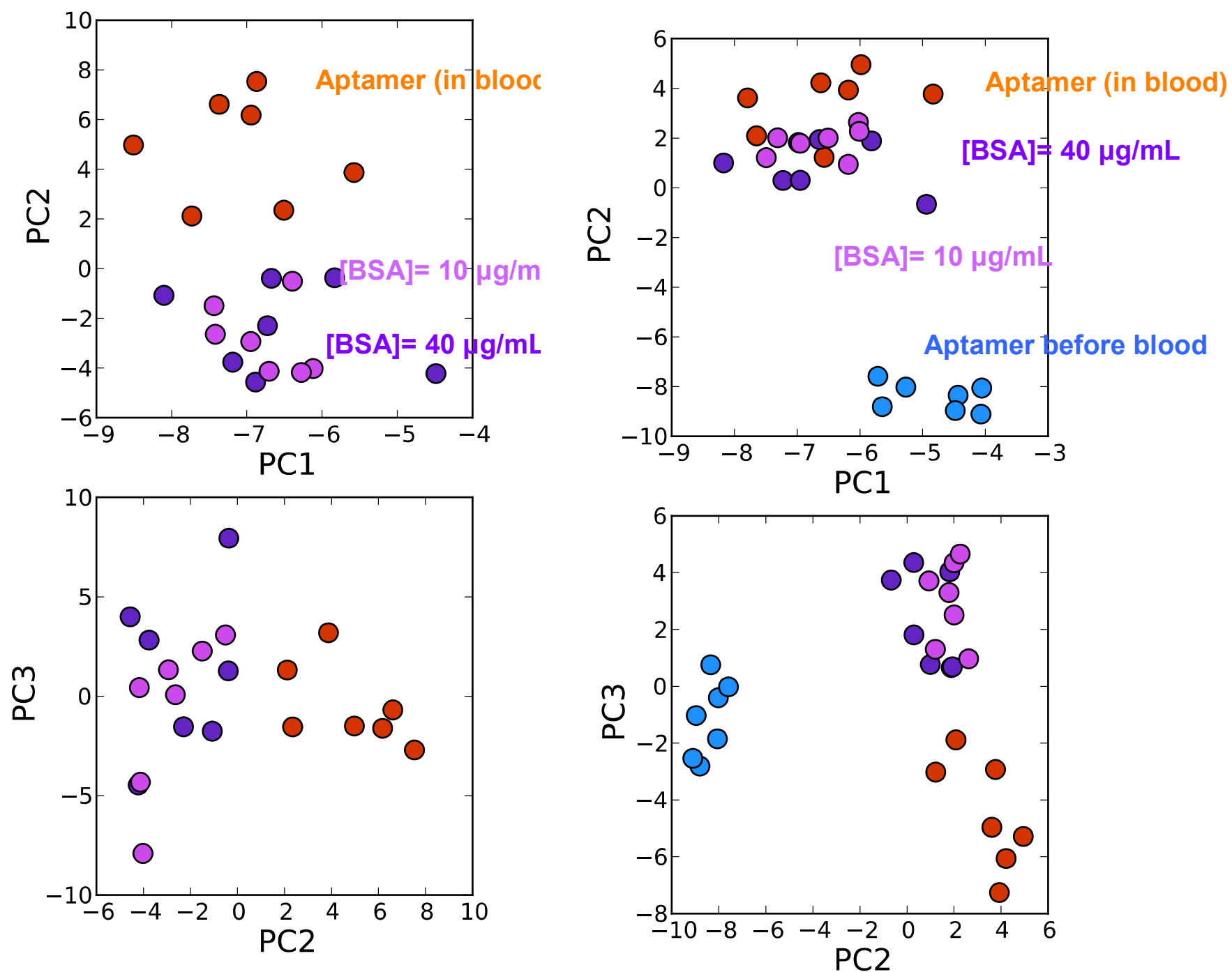

Figure S8. PCA plots showing that the spectra of BSA-exposed aptamer-modified AgFONs cannot be spectrally separated from the aptamer-modified AgFONs unexposed to BSA. 


\section{Effects of temperature, $\mathrm{pH}$ and ionic strength on AgFONs with benzenethiol}

\section{and $\mathrm{RBC}$ aptamer.}

Benzenethiol (BT) was employed as a model molecule to study the stability of the thiolate-silver interaction as well as the stability of the AgFONs themselves. While there is much precedent work exploring the thiolate-Au dissociation, there are no systematic studies of the stability of thiolate-silver interactions. ${ }^{4-6}$

The temperature influence on BT adsorbed to AgFON was studied first. As shown in Figure S9, the Raman bands at 1073, 1024, 1001, and $695 \mathrm{~cm}^{-1}$ shift are attributable to benzenethiol while the $1349 \mathrm{~cm}^{-1}$ shift peak is due to residual ethanol. As one might predict, higher temperature accelerated the dissociation of the S-Ag conjugation. Taking the intensity of the peak at $1073 \mathrm{~cm}^{-1}$ shift as an example (other benzenethiol peaks followed the same trend), the peak intensity decreased with increasing temperature. After the thermal treatment at $95{ }^{\circ} \mathrm{C}$, a significant decrease in the SERS signal was revealed a by two-tailed t-test ( $\mathrm{p}$ value after $30 \mathrm{~s}$ treatment is 0.0006 and after 5 min treatment is $\left.6 \times 10^{-6}\right)$. In addition, incubation at $70{ }^{\circ} \mathrm{C}$ for $30 \mathrm{~s}$ did not impact the SERS of benzenethiol ( $p$ value $=0.93$ ) while the SERS signal change at 70 ${ }^{\circ} \mathrm{C}$ for the 5 minute incubation was significant $(\mathrm{p}$ value $=0.01)$, indicating that the longer incubation time enhanced the dissociation of benzenethiol from AgFONs. The post-thermal treatment $\mathrm{AgFONs}$ were reincubated in the benzenethiol stock solution and then analyzed by SERS again. The results showed that the AgFON treated at a lower temperature and for a shorter time period recovered the initial SERS 
benzenethiol signals following reincubation. In contrast, the AgFONs that were treated at the highest temperature did not recover the benzenethiol SERS spectra following reincubation. This experiment demonstrates that the SERS signal decay at high temperature includes some contribution of permanent damage to the AgFON nanostructure.

In addition to the temperature excursion experiments detailed in the main text of the manuscript, both excursions in $\mathrm{pH}$ and ionic strength were also considered to induce reversible binding of ricin $B$ chain to the aptamer-modified substrate. The experiments were first done on benzenethiol-modified the AgFONs to test the stability of Ag-S bond in different $\mathrm{pH}$ and high ionic strength solutions. $\mathrm{pH}$ was varied within biologically relevant range: $5 \sim 8$. The ionic strength was adjusted to $1 \mathrm{M}$ using $\mathrm{NaCl}$. In all these conditions, the benzenethiol signal was unaffected, indicating that the Ag-S bond is stable in these conditions (Figure S9). The same experiments were performed on substrates with RBC captured on aptamer-modified AgFONs to test whether RBC would be released in these conditions. As with the temperature excursions, the Raman signatures of RBC binding remained despite the treatments conditions (Figure S10). 
a

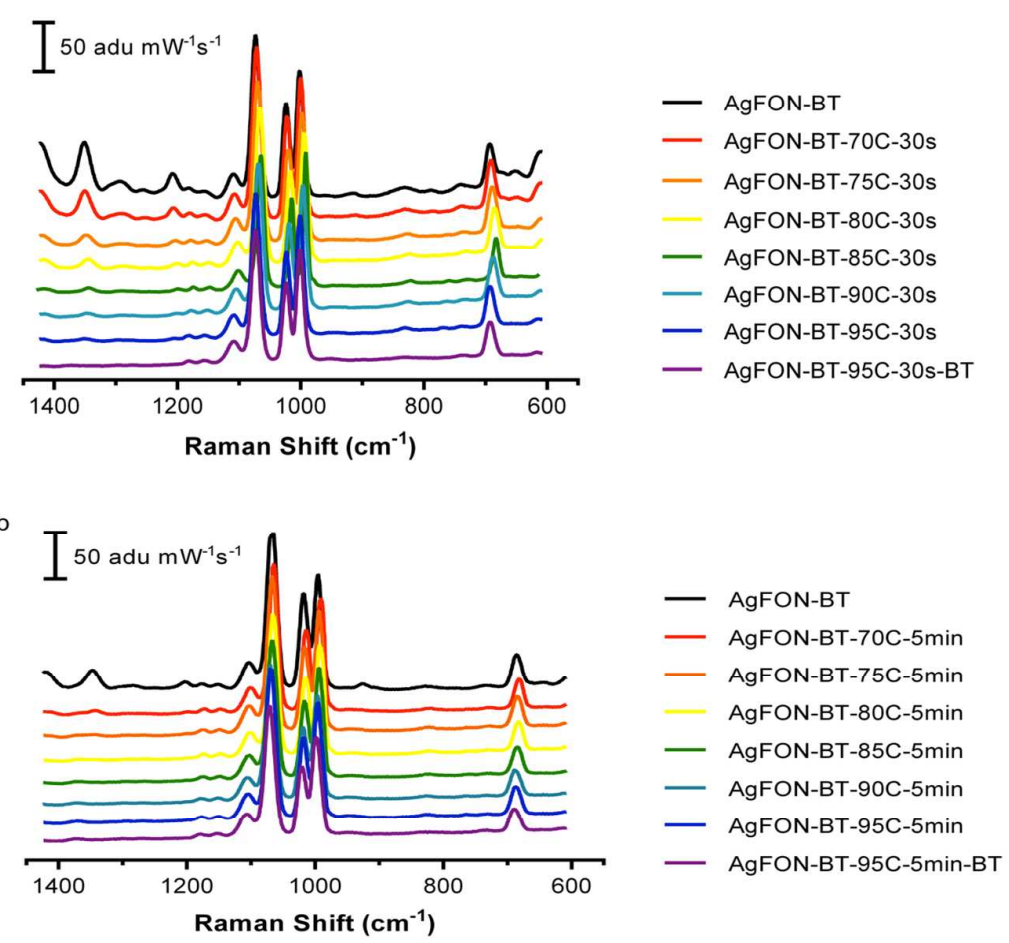

c

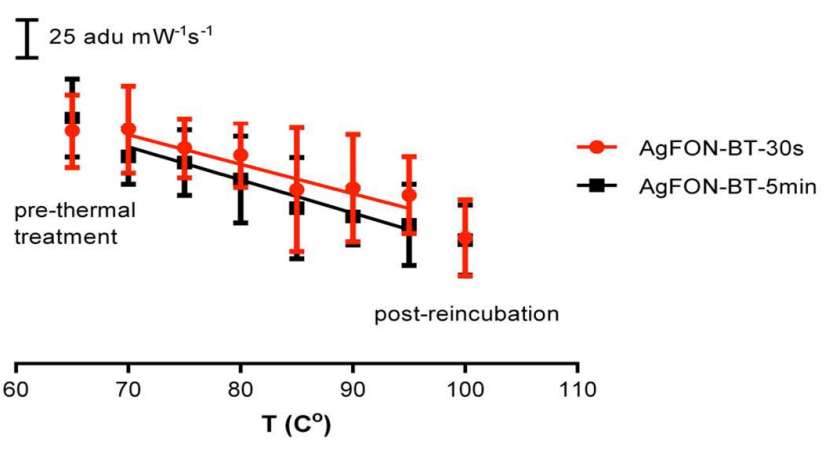

Figure S9. SERS of benzenethiol on AgFONs treated at elevated temperature for a) $30 \mathrm{~s}$ or b) 5 $\min$. The spectra are vertically off-set for clarity, $\lambda_{\mathrm{ex}}=632.8 \mathrm{~nm}$. Each trace is the average of 10 randomly chosen spots on the AgFON c) shows benzenethiol SERS intensity at $1071 \mathrm{~cm}^{-1}$ shift decreasing with increasing temperature, the far left spots are the intensities before the thermal treatments, the far right spots are the intensities after the thermally treated AgFONs were reincubated with benzenethiol stock solution (the purple spectra in both (a) and (b)). 

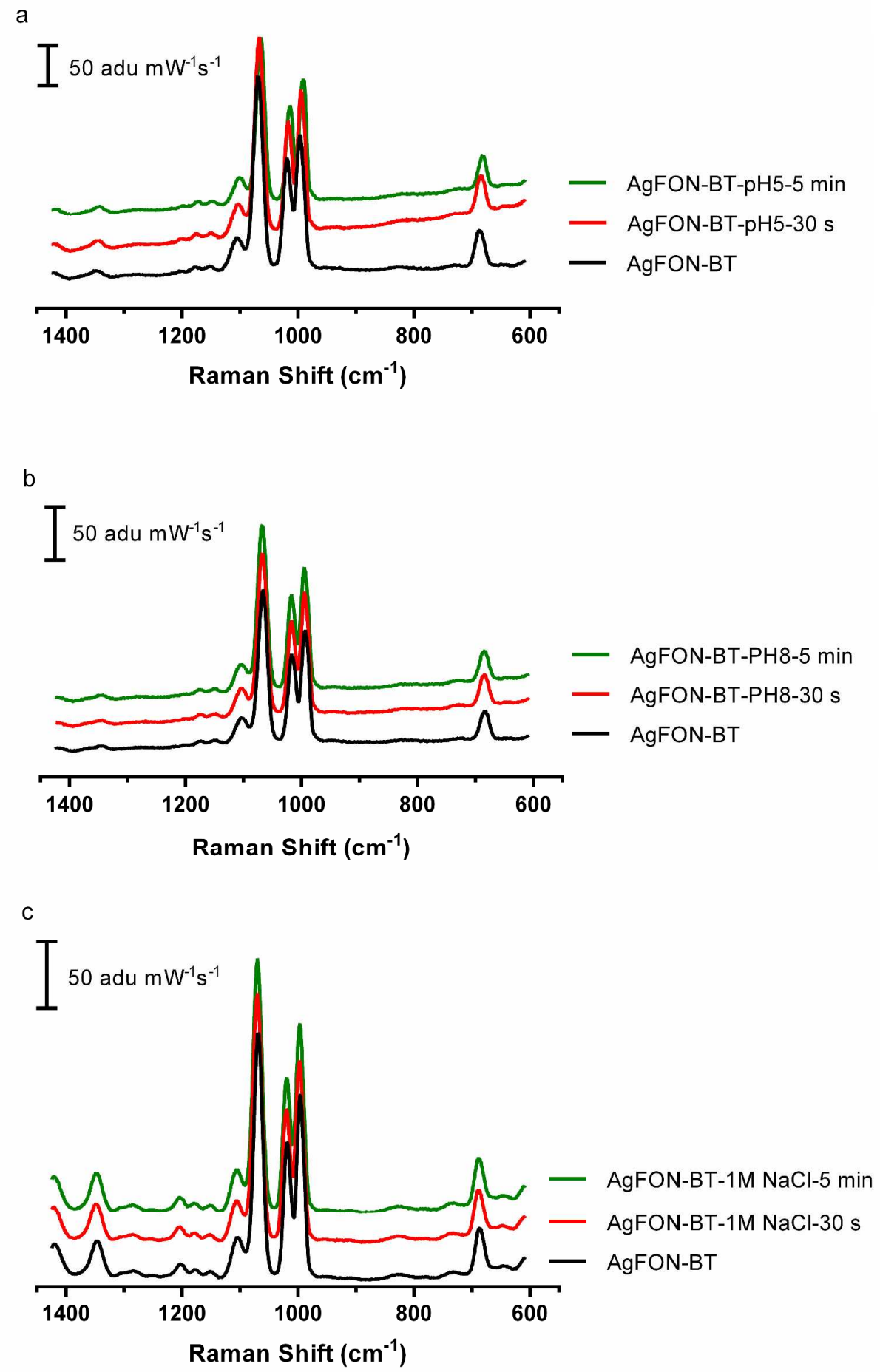

Figure S10. SERS of benzenethiol on AgFONs following solution condition excursion to a) $\mathrm{pH}$ of $5, \mathrm{~b}$ ) $\mathrm{pH}$ o f8, and c) ionic strength of $1 \mathrm{M} \mathrm{NaCl}$. Spectra are vertically offset to ease visualization. 

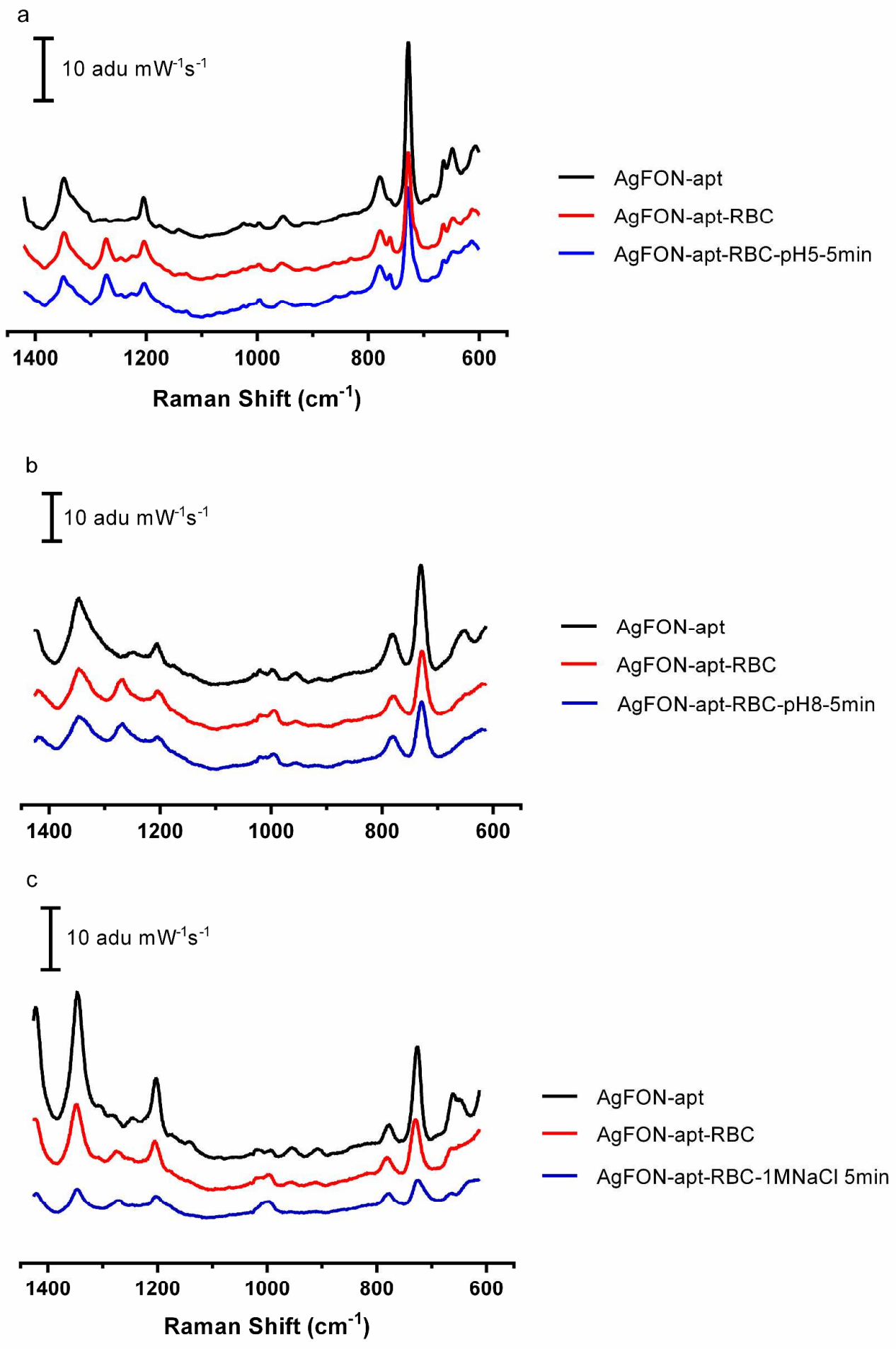

Figure S11. SERS of aptamer- and RBC-aptamer-modified AgFONs following solution condition excursion to a) $\mathrm{pH}$ of $5, \mathrm{~b}$ ) $\mathrm{pH}$ of 8 , and c) ionic strength of $1 \mathrm{M}$ $\mathrm{NaCl}$. Spectra are vertically offset to ease visualization. 


\section{References}

(1) Lamont, E. A.; He, L.; Warriner, K.; Labuza, T. P.; Sreevatsan, S. A Single DNA Aptamer Functions as a Biosensor for Ricin. Analyst 2011, 136, 3884-3895.

(2) Dieringer, J. A.; McFarland, A. D.; Shah, N. C.; Stuart, D. A.; Whitney, A. V.; Yonzon, C. R.; Young, M. A.; Zhang, X.; Van Duyne, R. P. Introductory Lecturesurface Enhanced Raman Spectroscopy: New Materials, Concepts, Characterization Tools, and Applications. Faraday Discuss. 2006, 132, 9-26.

(3) Lee, O.-S.; Schatz, G. C. Molecular Dynamics Simulation of DNA-Functionalized Gold Nanoparticles. The Journal of Physical Chemistry C 2009, 113, 2316-2321.

(4) Levy, M.; Benaglia, A. E. The Influence of Temperature and Ph Upon the Rate of Denaturation of Ricin. J. Biol. Chem. 1950, 186, 829-847.

(5) He, X.; Lu, S.; Cheng, L. W.; Rasooly, R.; Carter, J. M. Effect of Food Matrices on the Biological Activity of Ricin. J. Food Prot. 2008, 71, 2053-2058.

(6) Schulmerich, M. V.; Dooley, K. A.; Morris, M. D.; Vanasse, T. M.; Goldstein, S. A. Transcutaneous Fiber Optic Raman Spectroscopy of Bone Using Annular Illumination and a Circular Array of Collection Fibers. J. Biomed. Opt. 2006, 11, 060502-060502-3. 